\title{
Numerical Simulation of Current Dependence on Well Widths in AlGaAs/GaAs/AlGaAs Double Barrier Diode Structures
}

\author{
A.H. REZVANI* \\ Department of Physics, Tarbiat Modarres University, Tehran, Iran \\ and \\ The Center for Theoretical Physics and Mathematics, AEOI \\ P.O. Box 14155-1339, Iran
}

(Received June 7, 2002; revised version November 14, 2002)

A numerical procedure based on the time-dependent Kohn-Sham equation with an improved boundary condition for the modeling double barrier resonant tunneling diode is presented. The dependence of current components on well widths in $\mathrm{AlGaAs} / \mathrm{GaAs} / \mathrm{AlGaAs}$ structure is studied. An oscillatory behavior was observed as the width of the well is changed. Our evaluation shows that this oscillation cannot attribute to the well-known oscillation at resonance state.

PACS numbers: 73.63.Hs, 71.15.Mb, 71.10.Ca

\section{Introduction}

The double barrier resonant tunneling diode (DBRTD) structures have been extensively studied since the pioneering work of Tsu and Esaki $[1,2]$. These structures promise many interesting device applications such as in fast switching elements, high-frequency oscillators, highly sensitive detectors [3] and allow to study problems, relating energy levels in quantum well and tunneling which are of general interest in condensed matter physics.

*e-mail: arezvani@seai.neda.net.ir 
In a DBRTD the $n$-type doped semiconducting layers outside the quantum-well heterostructure act as reservoirs consisting of many electrons. The coupling between the RTD and reservoirs provides dissipation and sustains a steady, nonequilibrium current flow [4]. Because of the resonant tunneling of electrons through RTD structures and the observation of negative differential resistance (NDR) in DBRTD structure at room temperature and resonant tunneling of holes [5] it has received special attention. The original picture of the RTD as a Fabry-Perot resonator for electrons is defined by Tsu and Esaki and is developed further by Keldish [6]. In this essentially one-dimensional approach, the resonant enhancement of the transmission coefficient occurs when the incident electron energy from reservoir coincides with the energy of the bottom of the subband in the well. On the other hand, as Meir and Wingreen [7] have pointed out the NDR in RTD can be explained solely due to the tunneling of electrons from the three-dimensional states in the reservoir to the $2 \mathrm{D}$ states in the well since the component of the electron momentum transverse to the direction of tunneling is conserved. Subsequently, the electrons leave the well by tunneling through the other barrier. The dynamic response of DBRTD has been studied by nonequilibrium Green function, density functional theory and other methods by using one aspect of tunneling as mentioned before. The effects of the many electrons in reservoirs are very important. There are several models for it. In nonequilibrium Green function theory, the effects of reservoirs' electrons is put into the system by adding terms of the reservoirs' electrons and the interaction of reservoirs' electrons with the system to the Hamiltonian of the double barrier quantum well [8]. Frensley, on the other hand used the Wigner distribution function approach to calculate the $I-V$ characteristics of an RTD [9]. In Frensley's approach, the reservoirs were replaced by a constant flux of electrons incident on the device from the boundaries. The equilibrium distribution of these electrons was given by Fermi function. The simulation was based on the time-dependent Schrödinger equation for noninteracting electrons and the improved boundary conditions [10] were employed.

Tsuchiya and Sakaki [11] investigated experimentally the electron transport in resonant tunneling diodes. They showed that the resonant tunneling current depends generally on such parameter as barrier width, barrier heights, well width and the impurity concentration in the reservoirs. They also showed that the increase in well width lowers the resonant level first, which leads to the increase in the effective barrier height for electrons. This rise of the effective barrier height causes the sharpening of the transmission coefficient around the resonant tunneling current. They showed experimentally that the current decreased systematically as well width increased.

In the RTD or quantum-effect devices, the wave nature of the current carrier is fundamental to device performance. Due to this, the wave picture approach is suitable for evaluation of these devices. Nakano et al. in their paper [12] and our previous paper [13] pointed out that the time-dependent density functional theory 
(TDDFT) with improved boundary provides a suitable framework for studying the DBRTD devices. The purpose of this paper is to investigate the variation of well width and their effect on the electron transport in DBRTD devices. Our model system is a double barrier resonant tunneling diode consisting of a quantum well layer of GaAs sandwiched between AlGaAs as barrier layers. The well layers have variable widths between $0.4 \mathrm{~nm}$ and $20 \mathrm{~nm}$. The barrier layers have constant width of $5 \mathrm{~nm}$ thickness. The conduction-band discontinuity between the well and barrier layers is taken to be $0.23 \mathrm{eV}$. Both sides of the heterostructure are attached to thick $n$-type doped GaAs layers. The thickness of these layers is $150 \mathrm{~nm}$. The contact region, which is positively charged with ionized donor, is replaced by a uniform positive background. The doping density is $10^{18} \mathrm{~cm}^{-3}$ and is denoted by $n_{\mathrm{D}}$.

\section{Theoretical model}

We have performed real-time simulations for DBRTD connected to many-electron reservoirs by using TDDFT [14]. In TDDFT, one connects $V(r, t)$, external potential, to the electron density $n(r, t)$ and concludes that the external potential is determined (within an additive time-dependent function) by $n(r, t)$. This is chief point of TDDFT, thus, the knowledge of $n(r, t)$ is sufficient to determine the wave function and property of many-electron system. It is assumed that there exists a set of density function, which comes from a set of potentials (i.e. $v$-representable). In TDDFT, the time evolution of the wave functions are given by the Kohn and Sham [15], the effect of the interaction between particle and external potential, particles with particles is reflected in the functional $U[n(r, t)]$ that depends on the density distribution. The time-dependent Kohn-Sham equation is

$$
\left\{\mathrm{i} \hbar \frac{\partial}{\partial t}+\frac{\hbar^{2}}{2 m} \nabla^{2}-U[n(r, t)]\right\} \psi(r, t)=0 .
$$

The functional $U[n(r, t)]$ consists of all functional potential energies such as the external potential, exchange potential, the Hartree potential and etc. In Geldart and Rasolt [16], the Hartree potential is

$$
u_{\mathrm{H}}(r, t)=12 \int \mathrm{d}^{D} r^{\prime} \frac{n\left(r^{\prime}, t\right)}{\left|r-r^{\prime}\right|}
$$

and the exchange correlation energy in local density approximation (LDA) is

$$
u_{\mathrm{xC}}^{\mathrm{LDA}}[n(r, t)]=\left.\frac{\mathrm{d}}{\mathrm{d} n}\left\{n \varepsilon_{\mathrm{xc}}[n(r)]\right\}\right|_{n=n(r)},
$$

where $\varepsilon_{\mathrm{xc}}[n(r)]$ is the exchange energy per particle for a uniform electron gas with density $n(r)$. In DBRTD devices, the bare mass of electron must be replaced with the effective mass of conduction electrons and this depends on the space. The potential functional $U$ consists of the energy of the conduction-band edge, Coulomb potential arises from positive background charge in doped layers, the potential 
drop due to an external electric field, Hartree interaction and the exchange correlation energy per particle. The exchange correlation energy per particle is taken from the Green function Monte Carlo simulation results of Ceperley and Alder as parameterized by Vosko et al. [17]. Assuming translational invariance parallel to the interface, the electronic orbitals are products of wave functions, $\psi_{l}(r, t)$, parallel and perpendicular to the interface, respectively. The time evolution of the perpendicular wave is given by

$$
\left\{\mathrm{i} \hbar \frac{\partial}{\partial t}+\frac{\hbar^{2}}{2} \frac{\partial}{\partial x} \frac{1}{m^{*}(x)} \frac{\partial}{\partial x}-u[n(x, t)]\right\} \psi_{l}(x, t)=0 .
$$

In writing Eq. (4), the Ben Daniel and Duke Hamiltonian [18] is used. The electron density $n(r, t)$ is calculated from $n(r, t)=\sum_{i=1}^{N}\left|\psi_{i}(r, t)\right|^{2}$. The $n(r, t)$ integrated along interface surface, the electron density function along $x$-axis is obtained

$$
n(x, t)=\frac{m^{*}(x)}{\pi \hbar^{2}} \sum_{l=1}^{N}\left(\varepsilon_{\mathrm{F}}-\varepsilon_{l}\right)\left|\psi_{l}(x, t)\right|^{2},
$$

where $\varepsilon_{\mathrm{F}}$ and $\varepsilon_{l}$ are the Fermi and the subband energy, respectively, $N$ is the number of occupied levels. Because of homogeneity of system along interface surface the Hartree potential along the $x$-axis is

$$
u_{\mathrm{H}}(x, t)=\frac{2 \pi e^{2}}{\varepsilon} \int \mathrm{d} x^{\prime}\left|x-x^{\prime}\right| n\left(x^{\prime}, t\right) .
$$

For small increment of time ( $\Delta t$ ), formally, the solution of Eq. (1) can be written as

$$
\Psi_{l}(x, t+\Delta t)=T \exp \left(\int_{t}^{t+\Delta t} H(x, t)\right) \Psi_{l}(x, t)
$$

In our analysis, a time-dependent self-consistent method is used and Eq. (7) self-consistently with Eqs. (5) and (6) is solved. To execute Eq. (7), the partial differential operator is replaced by difference representation. The wave function and the potential energy are expressed on a regular spatial grid extending over both reservoirs and RTD. With using Nakano et al. methods, Eq. (7) transform to the set of linear algebraic equations. For boundary of system, we use the improved boundary condition treatment. We know the external potential is screened on the edge of the system which is called the asymptotic region according to some authors. Due to this, the external potential is taken to be constant in that region. Thus, $V_{\mathrm{L}}(t)$ and $V_{\mathrm{R}}(t)$, the external potentials on the left and right side asymptotic region are taken to be 0 and $V(t)$. On these regions the wave functions are scattering waves and are

$$
\Psi_{k}(x, t)=\exp \left(-\mathrm{i} \frac{E_{k} t}{\hbar}\right) \times \begin{cases}A_{k} \mathrm{e}^{\mathrm{i} k x}+B_{k}(x, t) \mathrm{e}^{-\mathrm{i} k x} & \text { for } x \leq x_{\mathrm{L}} \\ C_{k^{\prime}}(x, t) \mathrm{e}^{\mathrm{i} k^{\prime}(t) x} & \text { for } x \geq x_{\mathrm{R}}\end{cases}
$$

where $x_{\mathrm{L}}$ and $x_{\mathrm{R}}$ are the utmost points on the left and right asymptotic regions, respectively, $k$ and $k^{\prime}$ are the wave vectors, the coefficient $A$ is the constant am- 
plitude of incident waves, $B(x, t)$ and $C(x, t)$ are the amplitude of reflection and transmission which vary with space and time. $E_{k}$ is the energy of incident wave and is equal to

$$
E_{k}=\frac{(\hbar k)^{2}}{2 m^{*}}=\frac{\left[\hbar k^{\prime}(t)\right]^{2}}{2 m^{*}}+V_{\mathrm{R}}(t)
$$

Device Hamiltonian on the asymptotic region is

$$
H(x, t)=-\frac{\hbar^{2}}{2 m^{*}} \frac{\partial^{2}}{\partial x^{2}}+V_{\mathrm{R}}(t) .
$$

Since the space and density are homogeneous on the asymptotic region, the interactions between particles and particles with ambient space are put to the system by renormalization of the energy by a constant term. By using Eqs. (1) and (8), the time evolution of wave function on the left hand side asymptotic region with incident at left is

$$
\frac{\partial \Psi_{k}(x, t)}{\partial t}=-\frac{\mathrm{i}}{\hbar} E_{k}(x, t) \Psi_{k}(x, t)+\Gamma_{k}(x, t) \exp \left(-\mathrm{i} \frac{E_{k} t}{\hbar}\right),
$$

where $\Gamma_{k}$ is

$$
\Gamma_{k}(x, t)=\left[\frac{\hbar k}{m^{*}} \frac{\partial B(x, t)}{\partial x}+\frac{\mathrm{i} \hbar}{2 m^{*}} \frac{\partial^{2} B(x, t)}{\partial x^{2}}\right] \mathrm{e}^{-\mathrm{i} k x} .
$$

To solve the evolution of the wave function at the boundary, the partial differential with respect to time and the space in Eq. (11) is replaced by a difference representation, with much calculation and rearranging them, the following equation is obtained:

$$
\begin{aligned}
& \Psi_{k}\left(x_{1}, t+\delta t\right)\left[\exp \left(\frac{\mathrm{i} E_{k} \delta t}{2 \hbar}\right)+\frac{\hbar k \delta t}{2 m^{*} \delta x}+\frac{\mathrm{i} \hbar \delta t}{2 m^{*} \delta x^{2}}-\left(1+\frac{\mathrm{i} \hbar \delta t}{2 m^{*} \delta x^{2}}\right) \mathrm{e}^{-\mathrm{i} k \delta x}\right] \\
& =\Psi_{k}\left(x_{1}+\delta x, t+\delta t\right)\left[\left(\frac{\mathrm{i} \hbar \delta t}{4 m^{*} \delta x^{2}}+\frac{\hbar k \delta t}{2 m^{*} \delta x^{2}}\right) \mathrm{e}^{\mathrm{i} k \delta x}-\frac{\mathrm{i} \hbar \delta t}{4 m^{*} \delta x^{2}} \mathrm{e}^{-\mathrm{i} k \delta x}\right] \\
& +\Psi_{k}\left(x_{1}, t\right)\left[\exp \left(-\frac{\mathrm{i} E_{k} \delta t}{2 \hbar}\right)-\frac{\hbar k \delta t}{2 m^{*} \delta x}-\frac{\mathrm{i} \hbar \delta t}{2 m^{*} \delta x^{2}}-\left(1-\frac{\mathrm{i} \hbar \delta t}{2 m^{*} \delta x^{2}}\right) \mathrm{e}^{-\mathrm{i} k \delta x}\right] \\
& +\Psi_{k}\left(x_{1}+\delta x, t\right)\left[\left(\frac{\mathrm{i} \hbar \delta t}{4 m^{*} \delta x^{2}}+\frac{\hbar k \delta t}{2 m^{*} \delta x^{2}}\right) \mathrm{e}^{\mathrm{i} k \delta x}-\frac{\mathrm{i} \hbar \delta t}{4 m^{*} \delta x^{2}} \mathrm{e}^{-\mathrm{i} k \delta x}\right] \\
& +A \mathrm{e}^{\mathrm{i} k x_{1}}\left[\exp \left(\frac{\mathrm{i} E_{k} t}{2 \hbar}\right)+\exp \left(-\frac{\mathrm{i} E_{k} t}{2 \hbar}\right)\right] \\
& \times\left[\frac{\hbar k \delta t}{2 m^{*} \delta x}\left(1-\mathrm{e}^{\mathrm{i} 2 k \delta x}\right)-\frac{\hbar k \delta t}{2 m^{*} \delta x^{2}}\left(\mathrm{e}^{\mathrm{i} 2 k \delta x}-2+\mathrm{e}^{-\mathrm{i} 2 k \delta x}\right)\right]
\end{aligned}
$$


where $\delta x$ is distance between grid points. Equation (13) represents the unknown boundary wave function at time $t+\delta t$ in terms of the wave functions at time $t$ and the wave function on one step interior point at the same time. The same procedure must be done for the right hand side or transmission wave. The finite difference method is employed to solve Eq. (4) subjected to derived boundary condition and initial condition - the ground state of the many-electron system in the absence of external electric field. The procedure outlined so far is repeated for the right incident waves and to estimate the characteristic of the system, the superposition of left and right incident wave must be taken into account.

\section{Results and discussions}

The RK4 [19] method is used, the wave function and potential profile of the system at the equilibrium are calculated.

Figure 1 shows the resulting potential curves for three wells. The two semitriangular wells that are represented in the figure are produced by the junction between doped and undoped materials of the reservoir and the barrier. When the external potential is applied to the system, the reservoirs should form dipole layers and the applied potential is screened inside the reservoirs. In the case of an electron gas model, the screening must be complete and consequently the potential inside the doped layers should be constant.

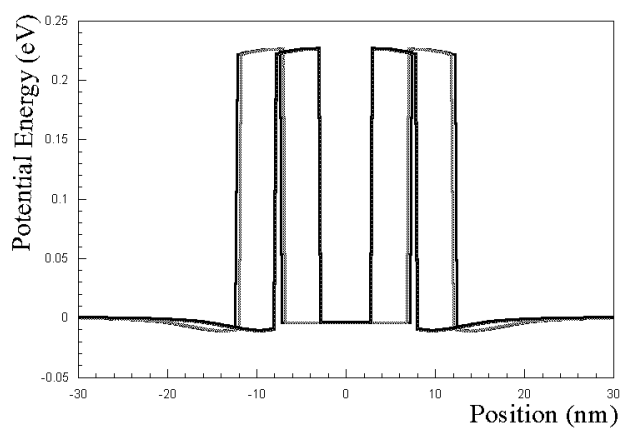

Fig. 1. The self-consistent calculated potential for ground state in the absence of electric field. The figure shows three potential curves for three quantum wells.

Figure 2 shows potential profile for three wells in nonequilibrium steady state achieved after $3000 \mathrm{fs}$. The result that is presented in the figure shows the screening effect inside the doped layers in three wells. It can be seen from the figure that the applied potential mostly drops in the active region. For calculation of the curves in Fig. 2, we first start with the equilibrium wave function and potential profile that is shown in Fig. 1, and then we switch on a uniform electric field and follow the real time evolution of the system. 




Fig. 2. The self-consistent potential profile for three quantum wells in the nonequilibrium steady state. The system is under external potential. The steady state is achieved after $3000 \mathrm{fs}$. The low variation of potential curve, on the edges of the system, shows the screening effect.

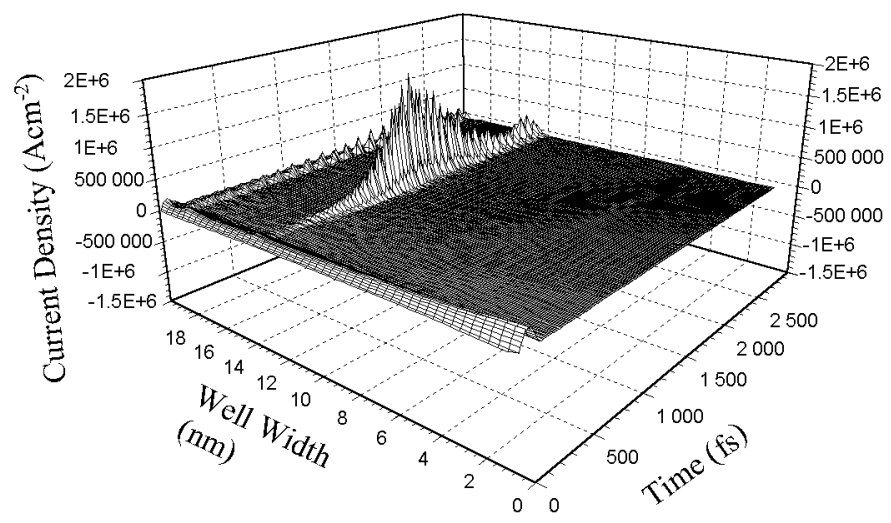

Fig. 3. The calculated average current over well and barriers width with respect to the well width and time. The applied potential is 0.08 volt. We repeated this calculation for the applied potentials $0.19,0.12$ volt and all results show this behavior on the well width of $14 \mathrm{~nm}$ and $18.4 \mathrm{~nm}$.

Figure 3 shows the time and wells width variation of the current density induced by an applied electric field after the field is switched on. The current density is calculated as an average over barrier and wells layers. Since the external source through boundary provides current carriers and many-electron reservoirs are the source of dissipation, a steady flow of current can be sustained for a long time. However, in the numerical analysis the large number of operation in long period of time and truncation errors deviate the calculated current density from true value. Figure 3 shows two oscillating currents at widths $14 \mathrm{~nm}$ and $18.4 \mathrm{~nm}$ after the transient diminishment. We cannot attribute this oscillation to the well-known os- 
cillation current that is observed in quantum well in resonant state. The well-known oscillation depends on the applied potential and this depends on the well width. We notify that the well-known oscillation also appears in the figure, but the large amplitude of oscillation observed by us overwhelmed it. We interpret the observed oscillation as follows. We know from the magneto-oscillation studies that the existence of space-charge build up in the quantum well at resonance regime and the theoretical investigation [20] exhibits that the space-charge build up depends on the current density through the system. In quantum well with one resonant level, when the Fermi energy level of many-electron system in emitter side close to the resonant level of the well, the current density and density of charge in the well increases. The build up of charge in the well rises the resonant level and then the current decreases. This is origin of the current oscillation in resonance regime in one level model. In our many-electron system, we have many electron states below the Fermi energy level. When the width of well increases, the number of resonant levels in the well increases and the resonant levels go to the down in the well. In this approach, we have large amplitude oscillating current when the large number of electron energy levels below the Fermi energy in the emitter side coincides with the energy of the resonant levels in the well and the currents of the each level are coherent. But in the system, the frequencies of oscillating currents of each level are approximately equal [20]. Because of this, the addition of these currents gives large amplitude current with a wave packet form. This current is represented in Fig. 4. Figure 4 shows the current versus time for three wells with different width. The well width increment in our analysis is $0.4 \mathrm{~nm}$. The figure shows the singular

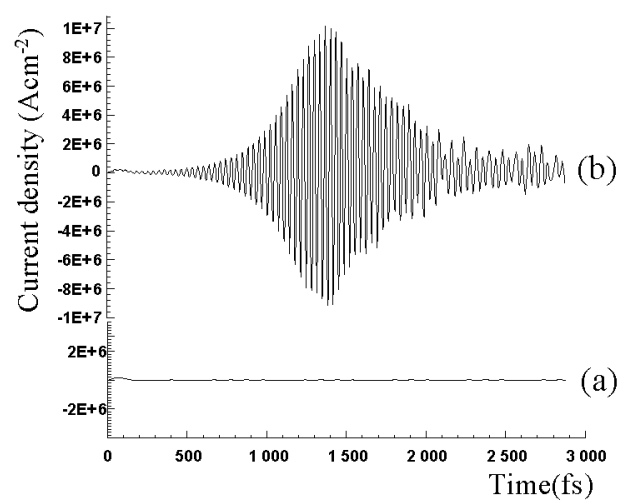

Fig. 4. The calculated average current over the well and barriers width with respect to time. Part (a) is for two wells with $13.6 \mathrm{~nm}$ and $14.4 \mathrm{~nm}$ width. The two curves are overlapped such that cannot be distinguished. Part (b) is for well with $14 \mathrm{~nm}$ widths. The oscillation current is such that it overwhelmed the transient current. This behavior is repeated along time axis. These figures show the singularity behavior in our numerical decision. 


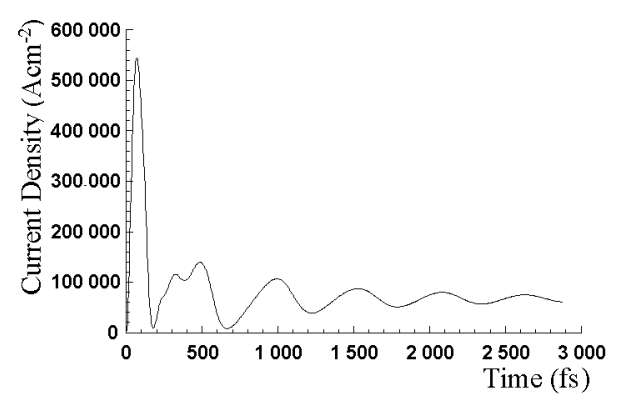

Fig. 5. The calculated average current over the well and barriers width with respect to time. The well width is $5.2 \mathrm{~nm}$. The applied potential is 0.19 volt. The oscillatory current on the steady state region is striking. The applied potential is about the resonant energy of this well.

behavior of this oscillating current with respect to variation of the well width at $14 \mathrm{~nm}$. The overlapping of curves for well width of $13.6 \mathrm{~nm}$ and $14.4 \mathrm{~nm}$, that is represented by part (a) in Fig. 4, is such that the two curves appear as one. Meanwhile, the figure displays sensibility of our observed oscillating current to the well width variation. The comparison between the oscillating current in Fig. 4 and the oscillating current in Fig. 5 that represent the well-known oscillating current curve, make appear the differences of the oscillating current observed by us and the well-known oscillating current.

\section{Conclusion}

We have used the GaAs-AlGaAs heterostructure due to its properties, namely its direct band gap and narrow energy band. The other property of GaAs - employed here - is its simple isotropic non-degenerate band extremum. It permits to use the envelope function model [21]. We have studied the current characteristic of double-barrier resonant tunneling diode connected to degenerate many-electron reservoirs and the interaction of the system with the external ambient space is simulated by using the improved open boundary condition treatment. The dynamics of the many-electron system of the reservoirs are explicitly taken into account by using the time-dependent density functional theory. We have previously used this method to calculate the density matrix and the phase correlation of carriers in well of $5 \mathrm{~nm}$ width. We showed that the outcome of this method is consistent with the experimental and the theoretical anticipations. Our result anticipates that RTD with the well width of about $14 \mathrm{~nm}$ and $18.4 \mathrm{~nm}$ reveal large amplitude oscillatory behaviors that is different from the observed current oscillation in RTD. 


\section{References}

[1] R. Tsu, L. Esaki, Appl. Phys. Lett. 22, 562 (1973).

[2] R. Tsu, L. Esaki, Appl. Phys. Lett. 24, 593 (1974).

[3] F. Capasso, K. Mohammed, A.Y. Cho, IEEE J. Quantum Electron. QE-22, 1853 (1986).

[4] R. Landauer, IBM J. Res. Dev. 1, 223 (1957); 32, 303 (1988).

[5] H. Ohnishi, T. Inata, S. Muto, N. Yokoyama, A. Shibatomi, Appl. Phys. Lett. 49, 1248 (1986).

[6] L.V. Keldish, Zh. Eksp. Teor. Fiz. 47, 1515 (1964) [Sov. Phys. JETP 20, 1018 (1965)].

[7] Y. Meir, N.S. Wingreen, Phys. Rev. Lett. 68, 2512 (1992).

[8] L.Y. Chen, C.S. Ting, Phys. Rev. Lett. 64, 3159 (1990).

[9] W.R. Frensley, Phys. Rev. Lett. 57, 2853 (1986).

[10] R.K. Mains, G.I. Haddad, J. Appl. Phys. 64, 3564 (1988); 67, 591 (1990).

[11] M. Tsuchiya, H. Sakaki, Appl. Phys. Lett. 49, 14 (1986).

[12] A. Nakano, P. Vashishta, R.K. Kalia, Phys. Rev. B 43, 11 (1991).

[13] A.H. Rezvani, M.A. Talebian, Iranian J. Phys. Res. 2, 5 (2000).

[14] E. Runge, E.K.U. Gross, Phys. Rev. Lett. 52, 997 (1984).

[15] W. Kohn, L.J. Sham, Phys. Rev. 136, B864 (1964).

[16] D.J.W. Geldart, M. Rasolt, In Strongly Correlated Electron Systems, Eds. M.P. Das, D. Neilson, Nova Science Publ., New York 1992.

[17] S.H. Vosco, L. Wilk, M. Nusair, Can. J. Phys. 58, 1200 (1980).

[18] W.H. Press, B.D. Flannery, S.A. Teakolky, W.T. Vetterling, Numerical Recipes in Fortran, Art of Scientific Computing, 2nd ed., Cambridge University Press, Cambridge 1992.

[19] T.L. Li, K.J. Kahn, J. Comput. Phys. 110, 292 (1994).

[20] V.J. Goldman, D.C. Tsui, J.E. Cunningham, Phys. Rev. Lett. 58, 1256 (1987).

[21] G. Bastard, Wave Mechanics Applied to Semiconductor Heterostructures, Halste Press, 1988. 